\title{
Testing and Improving the Dynamical Theory of Mass Exchange
}

\author{
Dmitry Bisikalo ${ }^{1}$ and Takuya Matsuda ${ }^{2}$ \\ ${ }^{1}$ Institute of Astronomy of the Russian Academy of Sciences, Moscow, Russia \\ email: bisikalo@inasan.ru \\ ${ }^{2}$ Department of Earth and Planetary Sciences, Kobe University, Kobe, Japan \\ email: tmatsuda312@yahoo.co.jp
}

\begin{abstract}
The study of the flow structure is of great importance, and the results can be used both for consideration of the evolutionary status of binary stars and for the interpretation of observational data. In this report we present the review of 3D gas dynamic models used for the description of the mass exchange in close binaries.

Main features of the flow structure in steady-state close binaries are summarized. It is shown that in self-consistent considerations the interaction between the stream from the inner Lagrangian point and the forming accretion disk is shock-free, and, hence, a "hot spot" does not form at the outer edge of the disk. To explain the presence of the observed zones of high luminosity in close binaries a self-consistent "hot line" model was proposed according to which the excess energy is released in a shock wave formed due to interaction between the circumdisk halo and the stream. The "hot line" model was confronted with observations and confirmed by virtue of comparison of synthetic and observational light curves for cataclysmic variables and by the analysis of Doppler tomograms.

The special attention is paid to the physics of accretion disks in binary systems and particularly to waves in disks. The possible observational manifestations of the "hot line" wave and two arms of the tidal shocks are discussed. We also suggest that an additional spiral density wave can exist in inner parts of the cold accretion disk. This spiral wave is due to the retrograde precession of flow lines in the binary system. The results of 3D gas dynamic simulation have shown that a considerable increase in the accretion rate (by an order of magnitude) is associated with the formation of the "precessional" spiral wave. Based on this fact we suggest a new mechanism for the superoutbursts and superhumps in close binaries.
\end{abstract}

Keywords. binaries: close, mass loss, accretion, accretion disks

\section{Introduction}

The overwhelming majority of stars (some researchers believe up to 80\%) are binaries. The presence of a gravity-connected companion of the star can affect the physical processes in the star and appreciably change its evolution. The fact is that belonging to a binary system limits the maximum size of the star. As early as 1848, E.A. Roche studied the motion of probe particles in the vicinity of a binary within a restricted problem of three bodies. He found that a certain space close to every component can be chosen, and within this space the gravity field of this component mainly affects the motion of a probe particle. In the course of the evolution of the star - a component of a binary the expansion out of the limits of this space which is now referred to as the Roche lobe results in mass loss of the star. A number of observations prove the complex flow structure in close binary stars (CBSs) caused by mass transfer in a system. Starting from the study of Struve (1941) who first conceived the idea of a gas stream appearing between components in $\beta$ Lyrae to explain the peculiar behaviour of the spectrum at eclipse, the 

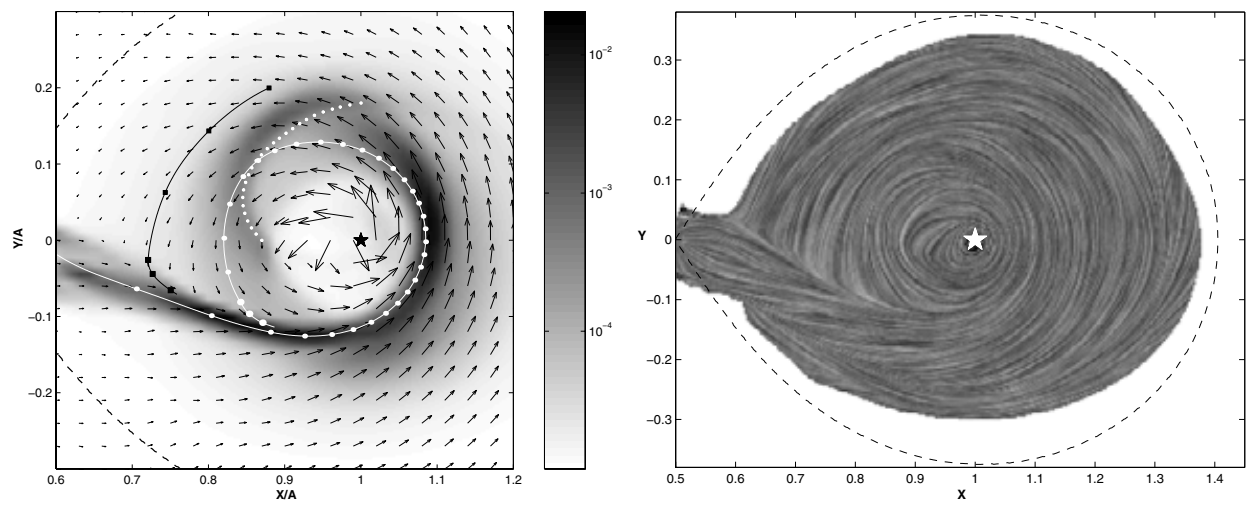

Figure 1. Left panel: The distribution of density over the equatorial plane. The $X$ and $Y$ coordinates are expressed in terms of the separation $A$. Arrows are the velocity vectors. The black asterisk is the accretor. The dashed line bounds the Roche lobe. The dotted line is the tidally induced spiral shock. Gas dynamic trajectory of a particle moving from $L_{1}$ to accretor is shown by a white line with circles. Another gas dynamic trajectory is shown by a black line with squares. Symbols of larger size correspond to passing of trajectory through shock wave located on the edge of the stream. Right panel: The visualization of the velocity vectors field using line integral convolution method. The $X$ and $Y$ coordinates are expressed in terms of the separation $A$. The white asterisk is the accretor, the dashed line is the Roche equipotential.

effects of mass transfer resulting in formation of gas flows, streams, disks, circumbinary envelopes and other structures were observed in a number of CBSs.

The main objective of this paper is the study of gas dynamics of mass transfer in CBSs. The study of the flow structure is of great importance, and the results can be used both for consideration of the evolutionary status and for the interpretation of observational data.

\section{Numerical modeling of mass transfer in close binaries}

To describe gas flows correctly, we should solve a complete system of gas dynamic equations. This system can be solved only within the framework of rather complex mathematical models. In the last fifteen years gas dynamics of mass transfer was numerically studied with the help of more realistic 3D models (see, e.g. pioneering works by Nagasawa et al., 1991; Hirose et al., 1991; Molteni et al., 1991). In this paper we summarize the results of 3D numerical simulation of mass transfer in semidetached binaries that were mainly obtained by Bisikalo et al. (1998 - 2006), Molteni et al. (2001), Harmanec et al. (2002), Boyarchuk et al. (2002), Fridman et al. (2003), Kaigorodov et al. (2006).

Analysis conducted in Bisikalo et al. (2003) has shown that for realistic values of parameters $\left(\dot{M} \simeq 10^{-12} \div 10^{-7} M_{\odot} \mathrm{y}^{-1}\right.$ and $\left.\alpha \simeq 10^{-1} \div 10^{-2}\right)$ the gas temperature in outer parts of the disk is between $\sim 10^{4} \mathrm{~K}$ and $\sim 2 \cdot 10^{5} \mathrm{~K}$. This implies that both hot and cold accretion disks can form in close binaries.

Let us consider the morphology of steady-state gaseous flows in a system with hot accretion disk. These solutions were obtained for temperatures of the outer parts of the accretion disk of $100000 \div 200000 \mathrm{~K}$.

The morphology of gaseous flows in considered binary system can be evaluated from Figure 1 (left panel). In this figure the distribution of density over the equatorial plane and velocity vectors are presented. We also show the gas dynamic trajectory of a particle moving from inner Lagrangian point $L_{1}$ to accretor (white line with circles) and the gas 


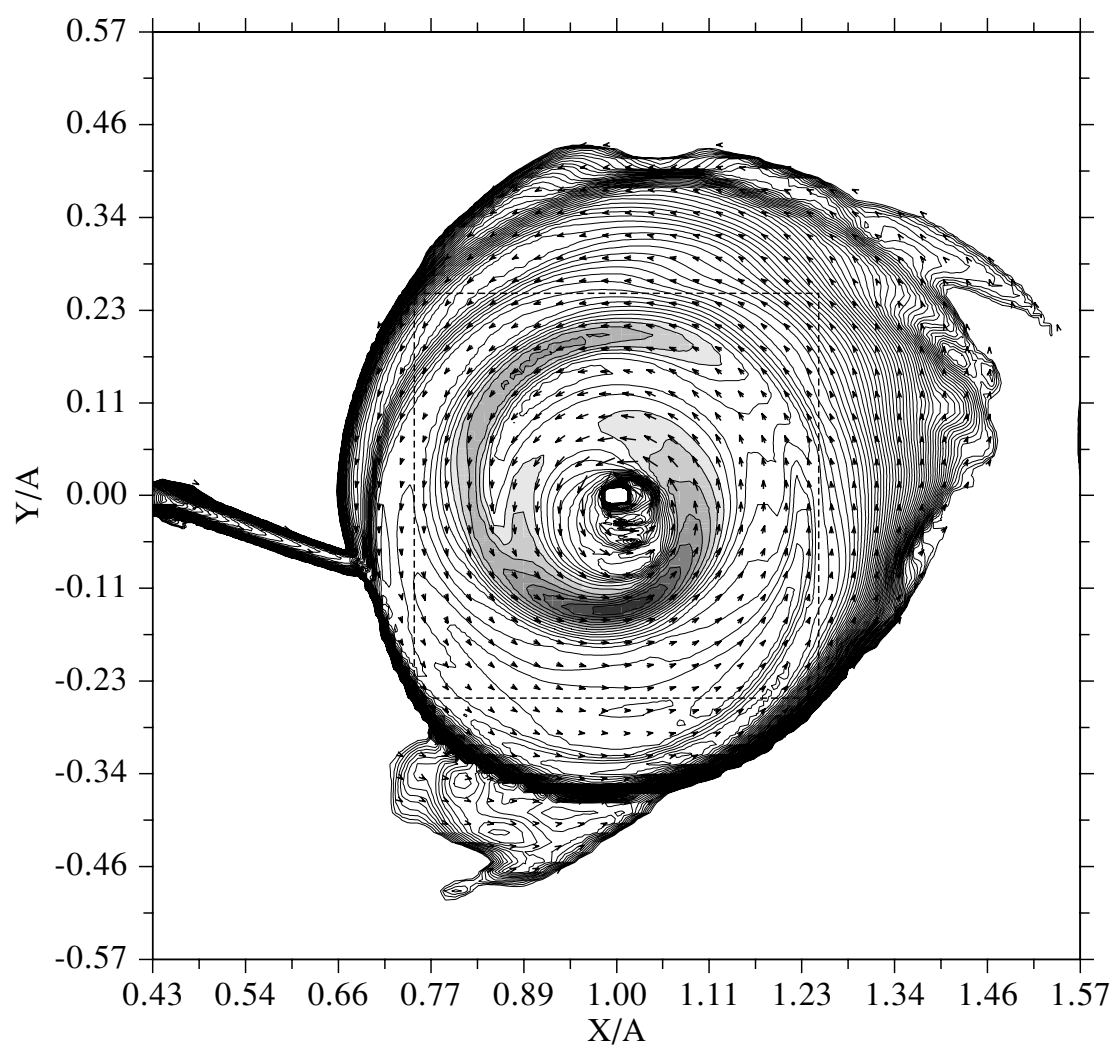

Figure 2. Contours of constant density and velocity vectors in the equatorial plane.

dynamic trajectory passing through the shock wave along the stream edge (black line with squares). The morphology of gaseous flows in semidetached binaries is governed by the stream of matter from $L_{1}$, quasi-elliptical accretion disk, circumdisk halo and circumbinary envelope. This classification of the main constituents is based on their physical properties: (i) if the motion of a gas particle is not determined by the gravitational field of accretor then this particle belongs to the circumbinary envelope filling the space between the components of binary; (ii) if a gas particle revolves around the accretor and after that mixes with the matter of the stream then it does not belong to the accretion disk but forms the circumdisk halo; (iii) the accretion disk is formed by the matter of the stream that is gravitationally captured by the accretor and hereinafter does not interact with the stream but moves to the accretor losing the angular momentum.

The analysis of the gas parameters along the flow lines reveals that the flow is smooth for all lines belonging to the disk up to the boundary flow line (white line with circles in Figure 1, left panel). The absence of breaks of the gas parameters suggests a shockfree interaction between the gas flow and the matter of the disk that in turn implies the absence of a "hot spot" on the edge of the disk. The uniform morphology of the flow results in the stream deflecting under the effect of the circumbinary envelope gas, approaches the disk along a tangent line and does not cause any shock perturbation of the disk edge. This fact is also obvious from the so-called texture figure (Figure 1, right panel) where the visualization of the velocity vector field using line integral convolution method is shown. At the same time analysis of the results proves that the interaction of 

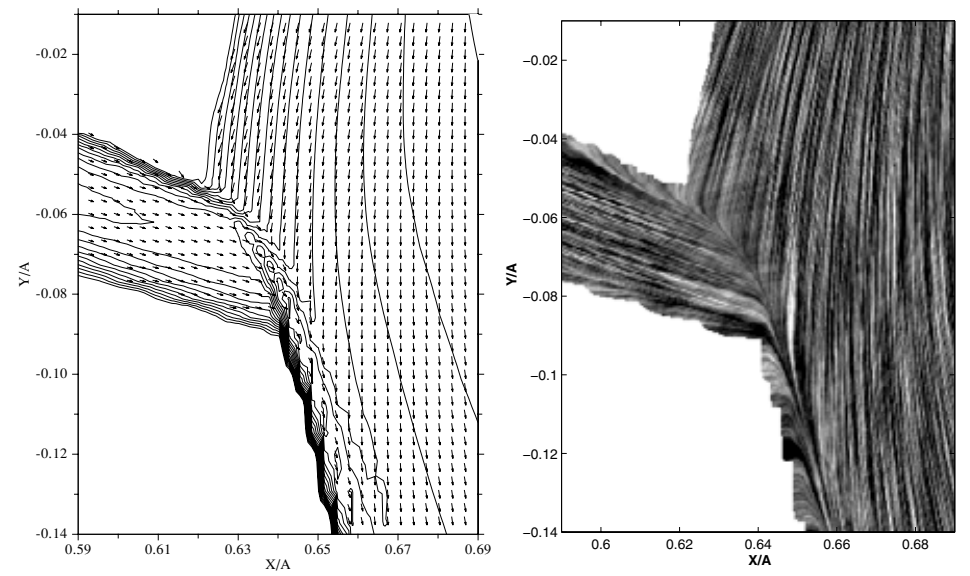

Figure 3. Contours of constant density and velocity vectors (left panel) and visualization of the velocity field (right panel) in the zone of interaction between the stream and disk.

a stream with the circumbinary envelope causes the formation an extended shock wave located along the stream edge. This shock is referred hereafter as "hot line".

From Figure 1 it is also seen that the spiral shock tidally induced by the donor-star appears in the disk. The position of this shock is shown in Figure 1 (left panel) by a white dotted line. Appearance of the tidally induced two-armed spiral shock was discovered by Sawada et al. (1986). Our 3D gas dynamic simulations for the "hot" case have shown only one-armed spiral shock while in the place where the second arm should be the flow structure is determined by the stream from $L_{1}$. It also should be stressed that in the "hot" case the spiral shock penetrates deeply to the inner part of the disk.

Let us consider the morphology of gaseous flows in a system with cold accretion disk. In the model used the temperature decreases to $\sim 1.4 \cdot 10^{4} \mathrm{~K}$ over the entire computation domain due to the radiative cooling. The basic problem here is whether the interaction between the stream and the disk remains shockless, as was shown for relatively hot disks. Figure 2 depicts density distribution and velocity vectors in the equatorial plane of the system (the $X Y$ plane). The shocks, which are formed in the disk, are seen as condensed isolines. The latter on the edge of the disk halo correspond to sharp decrease of density up to the background value. We can see the dense circular disk as well as the compact halo. The interaction of gas of the halo with the stream generates the shock outside the disk "hot line". The two-armed spiral shock wave forms in the disk. The both arms are located in the outer part of the disk.

Two panels of Figure 3 show density distribution and velocity vectors (left panel) and visualization of velocity field (right panel) in the zone of stream-disk interaction. Figure 3 shows that in the "cold" case the interaction between the circumdisk halo and the stream displays all features typical of an oblique collision of two streams. We can clearly see two shock waves and a tangential discontinuity between them. The gases forming the halo and stream pass through the shocks corresponding to their flows, mix, and move along the tangential discontinuity between the two shocks. Further, this material forms the disk itself, the halo, and the envelope.

Let us consider the changes occurring during the transition from the hot accretion disk to the cold one. The sketch of main peculiarities of the morphology of gaseous flows in semidetached binaries for "hot" and "cold" cases is given in Figure 4. These schemes are based on the results of 3D gas dynamic simulations. In Figure 4 the fragment of mass-losing star that fills its Roche lobe, the location of the inner Lagrangian point $L_{1}$, 

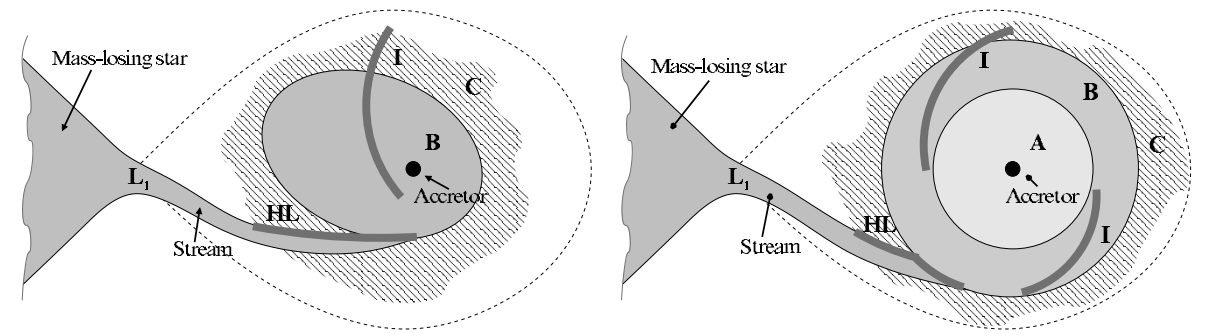

Figure 4. The sketch of main peculiarities of the morphology of gaseous flows in semidetached binaries for the case of high (left panel) and low (right panel) gas temperature.

the stream of matter from $L_{1}$, as well as the location of the accretor are shown. The dashed line marks the Roche lobe. The accretion disk is shown in Figure 4 as "A" and "B" zones, the near disk halo is shown as zone "C", the "hot line" is marked by "HL", and arms of tidal spiral shock are marked by "I". Our 3D gas dynamic simulations have shown that for the "cold" case when the radiative cooling decreases the gas temperature to $\sim 1.4 \cdot 10^{4} \mathrm{~K}$ the solution has the same qualitative features as that for the "hot" case, namely: the interaction between the stream and disk is shockless, the region of enhanced energy release is formed due to the interaction between the circumdisk halo and the stream and is located beyond the disk. The resulting shock - the "hot line" ("HL" in Figure 4) is fairly extended, that is particularly important for explaining the observations. However, unlike the solution with a high temperature in the outer regions of the disk, in the "cold" case, the shape of the zone of shock interaction between the stream and halo is more complex than a simple line. This is due to the sharp increase of the halo density as the disk is approached. Those parts of the halo that are far from the disk have low density, and the shock, due to their interaction with the stream, is situated along the edge of the stream. As the halo density increases, the shock bends, and eventually stretches along the edge of the disk. In the "cold" case the accretion disk (zones "A" and "B" in Figure 4) is significantly more dense as compared to the matter of the stream, the disk is thinner and has not quasi-elliptical but circular form. The size of the circumdisk halo is smaller as well. The second arm of the tidal spiral shock is formed, the both arms do not reach the accretor but are located in the outer part of the disk.

\section{The structure of the cold accretion disk}

Taking into account that the stream influences the dense inner part of the disk weakly as well as that all the shocks ("hot line" and two arms of tidal wave) are located in the outer part of the disk we can introduce a new element of flow structure for the "cold" case: the inner region of accretion disk (zone "A" in Figure 4) where the influence of gas dynamic perturbations mentioned above is negligible. Formation of non-perturbed region in the inner part of the disk allows to consider the latter as a slightly elliptical disk with the typical size of $\sim 0.2-0.3 A$ embedded in the gravitational field of binary. It is known (see, e.g., Warner, 1995), that the influence of companion star results in precession of orbits of particles rotating around of the binary's component. The precession is retrograde and its period increases with approaching the accretor.

For the accretion disk the orbits must be replaced by flow lines. Flow lines cannot intersect and can only touch by being tangent to each other. If the orbits precess such that the precession of distant flow lines tends to be faster, these distant flow lines will constantly overtake those with smaller semimajor axes. Since the flow lines in a disk 

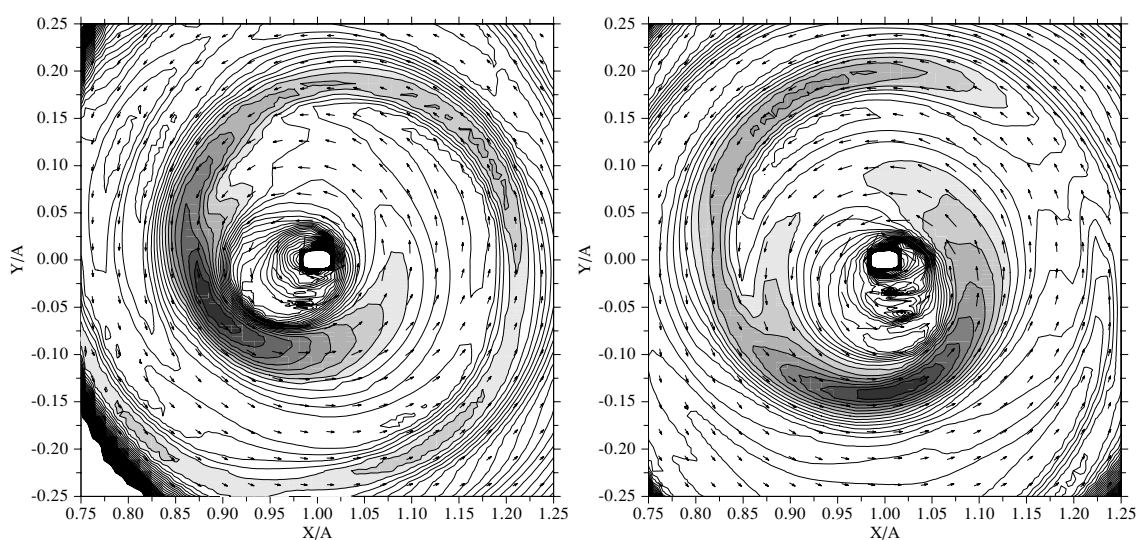

Figure 5. Density contours and velocity vectors in the inner part of the accretion disk for two moments of time. Enlarged image of the region delimited by the dashed curve in Figure 2.
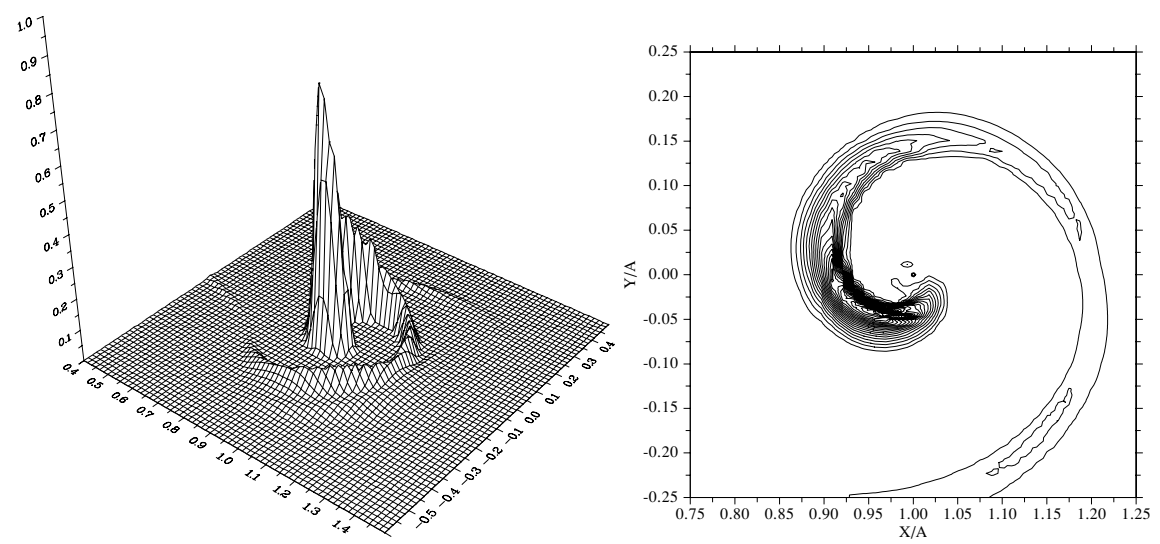

Figure 6. Distribution of the radial flux of matter in the equatorial plane of the disk. The flux is normalized to its maximum value.

cannot intersect, an equilibrium solution is established with time and all the flow lines begin to precess with the same angular velocity, i.e., to display rigid-body rotation. Let us consider a solution with the semimajor axes of the flow lines misaligned with respect to some chosen direction by an angle (turn angle) that is proportional to the semimajor axis of the orbit. It is obvious that such a solution should contain spiral structures. In particular, due to the nonuniformity of the motion along the flow line and the formation of a maximum density at apastron, the curve connecting the apastrons will form a spiral density wave. Indeed we have discovered (Bisikalo et al., 2004a) that the retrograde precession with specified law of precession rate results in formation of the density spiral wave of a new, "precessional" type in the inner part of the disk. This wave is clearly seen in Figure 2 in the region that was supposed to be unaffected by gas-dynamical perturbations. Figure 5 shows an enlarged view of the density distribution and velocity vectors in the inner part of the disk for two moments of time. Figure 5 shows the flows in a coordinate frame that rotates with the orbital period of the binary. The two-armed spiral wave is at rest in this coordinate system, as is quite natural for a tidal wave, while the inner spiral wave moves. Numerical results show that the "precessional" wave moves as a single entity, and its period is about ten times larger than the orbital one. Figure 6 shows the distribution of the radial flux of matter in the equatorial plane of the disk 

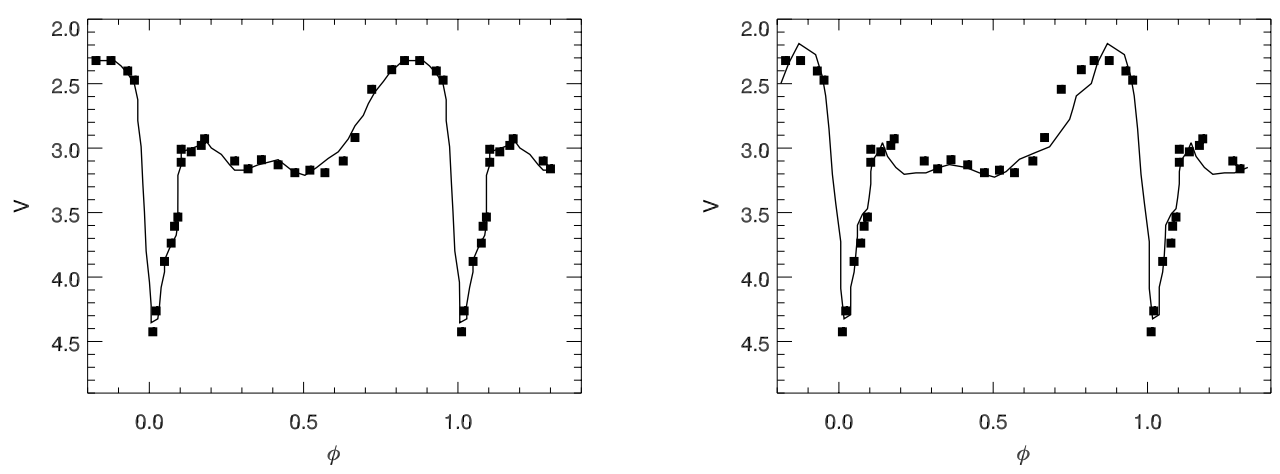

Figure 7. Mean light curve (points) of IP Peg in $V$ filter for quiescent state and synthetic (solid line) light curves obtained in the "hot line" (left panel) and in the "hot spot" (right panel) models.

in two ways; the radial flux is normalized to its maximum value. We can clearly see the spiral shape of the curve passing through the flux peaks. Due to the increase of the radial flux of matter behind the "precessional" density wave, the rate of accretion increases by approximately an order of magnitude compared to the wave-free solution.

Analysis of the results of a 3D gas dynamic simulation fully confirms the possibility of the generation of a spiral wave in the inner parts of a cold disk. The agreement between the qualitative analysis and the computational results makes us confident that the wave has a precessional origin.

\section{Astronomical applications of the "hot line" model}

\section{1. "Hot line" model versus "hot spot" model}

One of the best sources of information about the flow structure is the analysis of light curves of cataclysmic variables (CVs). The vast majority of light curves of normal CVs (U Gem type) display a so-called "orbital hump" around orbital phase $\sim 0.8$. Gorbatskii (1967) and Smak (1970) suggested that this is due to a "hot spot" at the edge of the accretion disk, where the disk collides with the stream from $L_{1}$. For many years, the "hot spot" model has been widely used to interpret light curves of cataclysmic binaries.

In terms of gas dynamics one can hardly understand the presence of a shock interaction between the flow and the steady-state accretion disk. Even if at the initial moment the stream from $L_{1}$ collides with the earlier-formed disk, in the course of time the morphology of the flow changes so that the stream-disk interaction has become shock-free, since the stream is the only source of matter for the disk. Three-dimensional simulations of gas dynamics of mass transfer prove that in a steady-state self-consistent solution the stream arrives at a tangent line to the disk, and the shock interaction of the disk and the stream (i.e., the standard "hot spot") does not appear. Meantime, the interaction of the circumbinary envelope with the stream was found to form an extended shock wave located along the edge of the stream. In spite of the fact that in this model the region of shock energy release is located outside of the accretion disk, there are good grounds to believe that this region ("hot line") can be considered in observation as an equivalent of a "hot spot" in the disk. In order to make certain that this assumption is valid and, hence, the gas dynamical model without a "hot spot" is adequate, we synthesize the light curves and compare them with observational data.

When applied to the interpretation of optical and infrared light curves of dwarf novae (Khruzina et al., 2001; 2003b), the "hot line" model shows certain advantages over the 

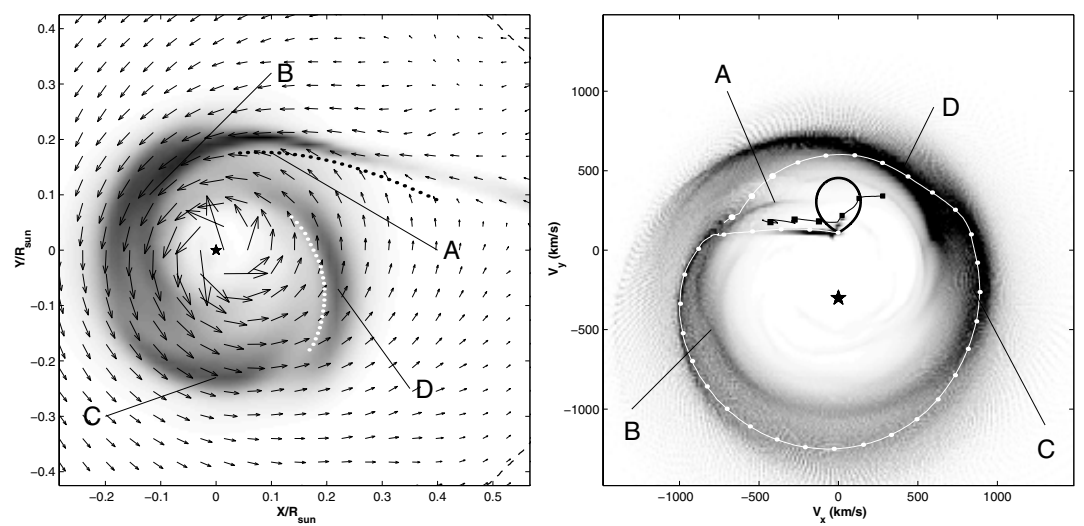

Figure 8. Left panel: Intensity map in the equatorial plane of the system in its quiescent state. A, B, C, and D denote zones with the highest intensity of radiation: A - "hot line", B - stream, $\mathrm{C}$-apogee of the quasi-elliptical disk, D - single arm of the tidal shock. Right panel: Synthetic Doppler tomogram for the system in its quiescent state. The Roche lobe of the donor star (thick black curve) and the accretor (star) are indicated. The white curve with circles and black curve with squares display gas dynamic trajectories in velocity space (see also Figure 1, left panel). $\mathrm{A}, \mathrm{B}, \mathrm{C}$, and $\mathrm{D}$ denote the same zones of maximum radiation intensity as in the left panel.

"hot spot" model (see Figure 7). Application of the "hot line" model to the light curves of the X-ray nova GU Mus (Khruzina et al., 2003a) demonstrated that this model is able to adequately describe the whole range of features of this X-ray nova's optical and IR orbital variability in quiescence. The "hot spot" model cannot reproduce the unusual shape of the light curve of GU Mus or, in some cases, the quiescent amplitude of the orbital light curves. Comparison between "hot spot" and "hot line" models for interpretation of the light curve of another X-ray nova in quiescence, XTE J1118+480 (Khruzina et al., 2005), has presented conclusive evidence in favor of the latter type of model.

The obtained results show that the "hot line" gas dynamic model can explain rather well the observed light curves of cataclysmic and X-ray binaries. Moreover, the use of the "hot line" model to interpret the light curves in systems where the application of standard models with the "spot" were unsuccessful, also results in rather good agreement of the synthetic and observed light curves.

\subsection{The model of superoutburst in binaries of $S U$ UMa type}

Superhumps are modulations of the light curves of binary systems with periods that differ from the orbital periods by several percent, and are observed mainly during superoutbursts in SU UMa systems. Currently, the most popular model explains these light variations as an effect of the precession of the accretion disk. The presence of the Lindblad $3: 1$ resonance in the disk results in an instability that leads to the precession of the outer part of the disk, with a period that is appreciably longer than the orbital period. The beating of the orbital and precessional periods gives rise to the periodic variations that are identified with superhumps. This model has several shortcomings, the most important being that it implies a limit on the maximum component mass ratio. In order for $3: 1$ resonance to be located inside the accretion disk, the ratio of the donor and accretor masses q must be lower than $\sim 0.33$, while there are several observed systems with superhumps where the mass ratio is well above the critical value. The estimates of the mass ratio for TV Col show that $q$ can be as much as $0.6 \div 0.9$ (Retter et al., 2003).

A new mechanism for the formation of superhumps in SU UMa systems was suggested in Bisikalo et al. (2004b). The basis of this mechanism is the idea that a precessional-type 

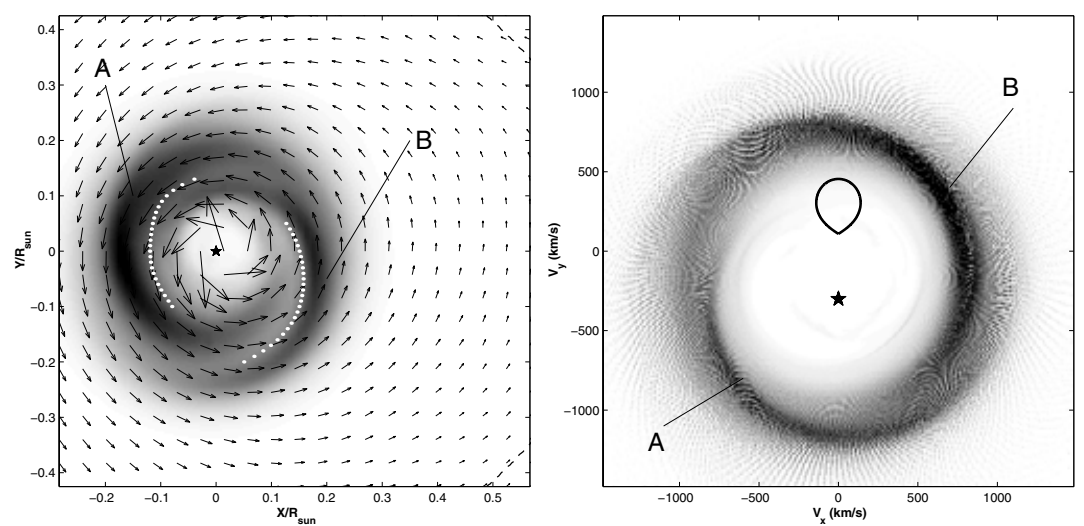

Figure 9. Left panel: Intensity map in the equatorial plane of the system in its active state. $\mathrm{A}$ and $\mathrm{B}$ denote zones with maximum intensity of radiation corresponding to the two arms of the tidal shock. The arrows denote velocity vectors in the laboratory coordinate system. The star marks the accretor. Right panel: Synthetic Doppler tomogram for the system in its active state. The Roche lobe of the donor star (thick black curve) and the accretor (star) are shown. $\mathrm{A}$ and $\mathrm{B}$ denote the same zones with maximum radiation as in the left panel.
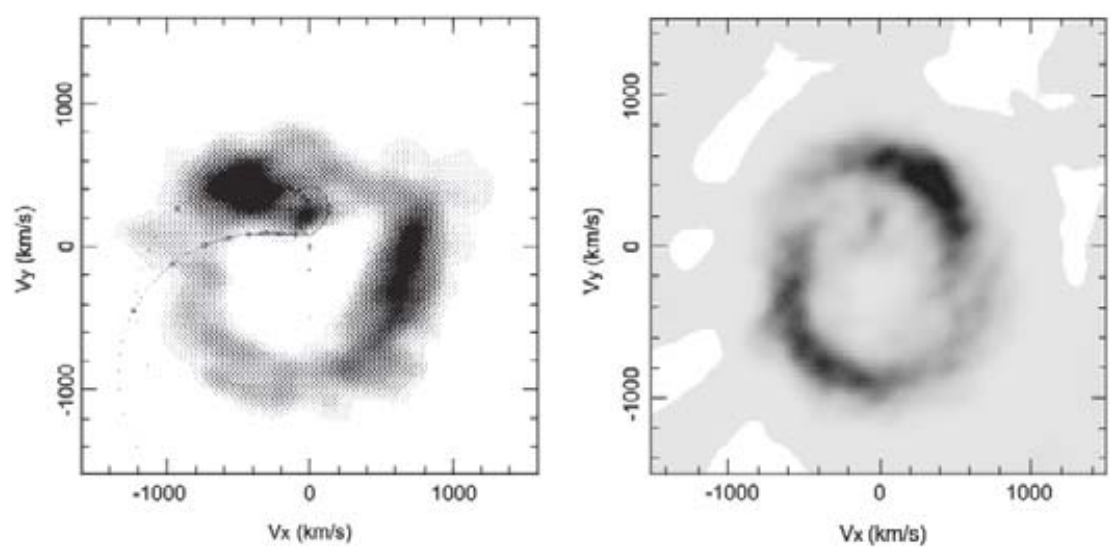

Figure 10. Doppler map of IP Peg for quiescent (left panel) and active (right panel) states of the system (Morales-Rueda et al., 2000; Wolf et al., 1998).

density wave can form in the cold accretion disk (see Section 3). After the formation of the precessional density wave in the disk, the rate of accretion grows sharply (by up to an order of magnitude). Matter approaches the surface of the accretor along the precessional wave, and the region of accretion is localized in azimuth, and, hence, forms a radiating spot at the surface of accretor. The increase in the accretion rate due to the density wave explains both the development of a superoutburst and the amplitude of the superhump (Bisikalo et al., 2004b, 2006; Kaigorodov et al., 2006). The beating of the orbital period and the precessional period of the wave results in the superhump period, which is slightly larger than the orbital period.

If outburst is associated with the formation of a "precessional" wave we will see the typical structure of the cold disk during the outburst (see Figure 4, right panel). On the other hand, being in the frame of this mechanism, during the quiescent state the disk can not have the spiral "precessional" wave. It means that in this state inner parts of the disk 


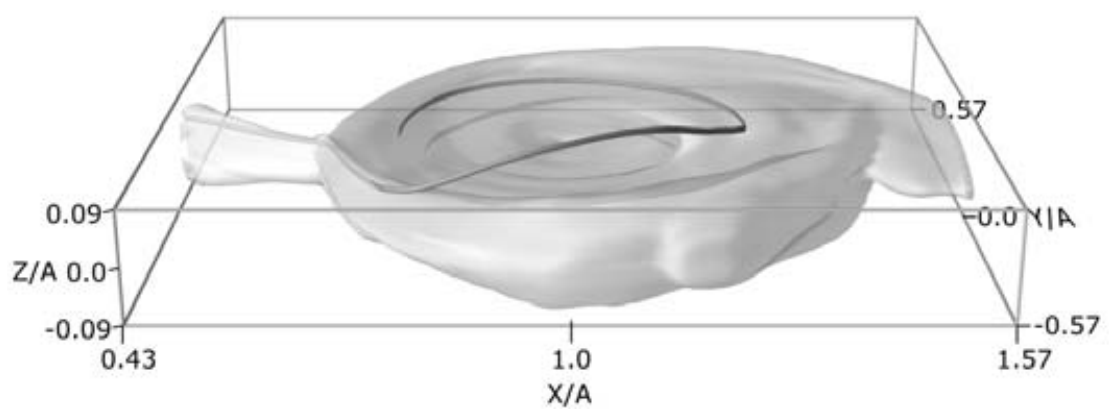

Figure 11. Three-dimensional surface of constant density and fragment of streamline emerging from the neighborhood of $L_{1}$.

are gasdynamically disturbed. At the beginning of the quiescent state the gas is heated up to the high temperature, so the solution will be close to one obtained for the hot disk (see Figure 4, left panel). Using 3D gas dynamic simulations alongside with the Doppler tomography technique allows to identify the main features of the flow on the Doppler maps without solution of an ill-posed inverse problem (Kuznetsov et al., 2003). Figures 8 and 9 show intensity maps and synthetic Doppler tomograms for system in quiescent and in outburst state. Comparison of the synthetic tomograms with observations (Figure 10) shows that, in the quiescent state of the system, the most luminous components are (i) the "hot line" (zone A), and (ii) the gas condensation at the apogee of the quasi-elliptical disk (zone C). Both the single arm of spiral shock wave (zone D) and the stream itself (zone B) contribute little to the luminosity. In the active state of the system, when the stream does not play an appreciable role and the disk dominates, both areas of enhanced luminosity in the observational tomograms are associated with the two arms of the spiral shock wave in the disk. Comparison of synthetic Doppler tomograms with observations shows that the considered model does not contradict observations.

This superoutburst model based on the presence of a precessional spiral wave in the accretion disk made it possible for the first time to explain all important observational manifestations of superoutbursts and superhumps in SU UMa systems, including their period, duration, and energy; the anticorrelation of the brightness and color temperature at the maximum of an ordinary superhump; the late-superhump phenomenon, etc. Moreover, this model does not place strict constraints on the component mass ratio, and so can be applied to the superhumps in systems of large mass ratio.

\subsection{On possible nature of pre-eclipse dips in light curves of semidetached systems with steady-state disks}

Observations of low-mass X-ray binaries (LMXBs) have revealed pre-eclipse dips in the $\mathrm{X}$-ray light curves for several systems. Similar light-curve features in various wavelength ranges have also been recorded for a number of cataclysmic binaries in outburst. Further observations showed that light curve dips can also appear when a system is in a stationary state. Studies of the ultraviolet light curves of the eclipsing nova-like cataclysmic binaries UX UMa and RW Tri (Mason et al., 1997 ) confirmed this result, and suggested that this phenomenon was universal in semi-detached binaries with accretion disks. It is interesting that, in contrast to the cataclysmic systems, systems with stationary disks display preeclipse dips at much earlier phases, $\sim 0.6 \div 0.7$ (Mason et al., 1997; Froning et al., 2003).

In order to explain pre-eclipse dips at phases $\sim 0.8$ in light curves of LMXBs and CVs, the idea that during the outburst the stream collides with the disk and ricochetes off the disk's edge is considered as the best way to explain the presence of matter at heights 
considerably exceeding the disk thickness (see, e.g., Armitage \& Livio, 1996). Shock-free nature of the interaction between the stream and the steady-state disk in the stationary solution poses the question on the cause of the of matter presence at significant height above the accretion disk in systems of this type. The results of 3D modeling have shown that in the absence of direct collision between the stream and the disk the formation of thickening of the halo above the disk is also possible (Bisikalo et al., 2005).

In the framework of the "hot line" model the significant part of the matter gets the acceleration in vertical direction. Gas movement in the vertical direction together with its movement along the outer edge of the disk leads to the gradual increase of the neardisk halo width. Maximum of the calculated thickening located above the outer part of the disk corresponds to the $\sim 0.7$ phase (see Figure 11) that is in agreement with the observed values for systems with steady-state disks. This fact confirms the "hot line" model suggested earlier for description of the flow structure in semi-detached binaries and gives new opportunities for the interpretation of the light curves of such systems.

\section{Conclusions}

Results of gas dynamic modeling allow us to understand better the structure of gaseous flows in close binaries. For systems with stationary accretion disks the flow structure is described by the "hot line" model that is in a good agreement with observations.

\section{Acknowledgements}

The work was supported by the Russian Foundation for Basic Research (projects nos. 05-02-16123, 05-02-17070, 05-02-17874, 06-02-16097), the Scientific Schools Program (grant no. NSh-4820.2006.2), and the basic research programs "Mathematical modeling and intellectual systems" and "Origin and evolution of stars and galaxies" of the RAS.

\section{References}

Armitage, P.J. \& Livio, M. 1996, ApJ 470, 1024

Bisikalo, D.V., Boyarchuk, A.A., Chechetkin, V.M., Kuznetsov, O.A., \& Molteni, D. 1998a, MNRAS, 300, 39

Bisikalo, D.V., Boyarchuk, A.A., Kuznetsov, O.A., Khruzina, T.S., \& Cherepashchuk, A.M. 1998b, Astron. Rep. 42, 33

Bisikalo, D.V., Boyarchuk, A.A., Kuznetsov, O.A., \& Chechetkin, V.M. 1999a, Astron. Rep. 43, 229

Bisikalo, D.V., Boyarchuk, A.A., Chechetkin, V.M., Kuznetsov, O.A., \& Molteni, D. 1999b, Astron. Rep. 43, 797

Bisikalo, D.V., Harmanec, P., Boyarchuk, A.A., Kuznetsov, O.A., \& Hadrava, P. 2000a, A\&A 353,1009

Bisikalo, D.V., Boyarchuk, A.A., Kuznetsov, O.A., \& Chechetkin, V.M. 2000a, Astron. Rep. 44, 26

Bisikalo, D.V., Boyarchuk, A.A., Kilpio, A.A., Kuznetsov, O.A., \& Chechetkin, V.M. 2001, Astron. Rep. 45, 611

Bisikalo, D.V., Boyarchuk, A.A., Kaigorodov P.V., \& Kuznetsov, O.A. 2003, Astron. Rep. 47, 809

Bisikalo, D.V., Boyarchuk, A.A., Kaigorodov P.V., Kuznetsov, O.A., \& Matsuda T. 2004a, Astron. Rep. 48, 449

Bisikalo, D.V., Boyarchuk, A.A., Kaigorodov P.V., Kuznetsov, O.A., \& Matsuda T. 2004b, Astron. Rep. 48, 588

Bisikalo, D.V. 2005, ApधSSS 296, 391

Bisikalo, D.V., Kaigorodov P.V., Boyarchuk, A.A., \& Kuznetsov, O.A. 2005, Astron. Rep. 49, 701 
Bisikalo, D.V., Boyarchuk, A.A., Kaigorodov P.V., Kuznetsov, O.A., \& Matsuda T. 2006, Chin. J. Astron. Astrophys., vol. 6, p. 159

Boyarchuk, A.A., Bisikalo, D.V., Kuznetsov, O.A., \& Chechetkin, V.M. 2002, Mass transfer in close binary stars, Taylor and Francis, London

Fridman, A. M., Boyarchuk, A. A., Bisikalo, D. V., Kuznetsov, O. A., Khoruzhii, O. V., Torgashin, Yu. M., \& Kilpio, A. A. 2003, Phys. Lett. A 317, 181

Froning, C.S., Long, K.S., \& Knigge, C. 2003, ApJ 584, 433

Gorbatskii V.G. 1967, Astrofisica 3, 245

Hirose, M., Osaki, Y., \& Mineshige, S. 1991, Publ. Astron. Soc. Japan, vol. 43, p. 809

Harmanec, P., Bisikalo, D. V., Boyarchuk, A. A., \& Kuznetsov, O. A. 2002, A\&̋A. 396, 937

Kaigorodov P.V., Bisikalo, D.V., Kuznetsov, O.A., \& Boyarchuk, A.A. 2006, Astron. Rep. 50, 537

Khruzina T.S., Cherepashchuk, A.M., Bisikalo, D.V., Boyarchuk, A.A., \& Kuznetsov O.A. 2001, Astron. Rep. 45, 538

Khruzina T.S., Cherepashchuk, A.M., Bisikalo, D.V., Boyarchuk, A.A., \& Kuznetsov O.A. 2003a, Astron. Rep. 47, 621

Khruzina T.S., Cherepashchuk, A.M., Bisikalo, D.V., Boyarchuk, A.A., \& Kuznetsov O.A. 2003a, Astron. Rep. 47, 848

Khruzina T.S., Cherepashchuk, A.M., Bisikalo, D.V., Boyarchuk, A.A., \& Kuznetsov O.A. 2005, Astron. Rep. 49, 79

Kuznetsov, O.A., Bisikalo, D.V., Boyarchuk, A.A., Khruzina, T.S., \& Cherepashchuk, A.M. 2001, Astron. Rep. 45, 872

Mason, K.O., Drew, J.E., \& Knigge, C. 1997, MNRAS 290, L23

Molteni, D., Belvedere, G., \& Lanzafame, G. 1991, MNRAS, 249, 748

Molteni, D., Kuznetsov, O. A., Bisikalo, D. V., \& Boyarchuk, A. A. 2001, MNRAS 327, 1103

Morales-Rueda, L., Marsh, T. R., \& Billington, I. 2000, MNRAS 313, 454

Nagasawa, M., Matsuda, T., \& Kuwahara, K. 1991, Numer. Astrophys. in Japan, vol. 2, p. 27

Retter, A., Hellier, C., Augusteijn, T., et al. 2003, MNRAS 340, 679

Sawada K., Matsuda T., \& Hachisu I. 1986, MNRAS 219, 75

Smak J. 1970, AcA 20, 312

Struve, O. 1941, ApJ 93, 104

Warner, B. 1995, Cataclysmic Variable Stars, Cambridge University Press, Cambridge

Wolf, S., Barwig, H., Bobinger, A., et al. 1998, A\&A 332, 984

\section{Discussion}

John SouthworTh: There are some very good light curves of eclipsing CV where a bright spot model fits well but not perfectly. Would you be able to improve upon this with your model?

BisikAlO: Hopefully, yes.

MERCEDES RICHARDS: What are the inner boundary conditions for your simulations (i.e., do your simulations allow you to follow the flow down to the star)?

Bisikalo: The inner boundary conditions is set close to the surface of the WD. Using the supercomputer allows us to consider the flow structure up to the surface of WD.

SlAVEK Rucinski: Can your hydrodynamic model be used for close main-sequence stars when one star fills the Roche lobe and sends the matter straight into the surface of the second star? We see many EB stars where one side is directly heated by the colliding stream (good example: V361 Lyrae). 
BISIKALO: Yes, this model is suitable for consideration of the flow structure in such systems. Actually we are now working on simulations of a system where streams collide directly onto the surface of the second star.

Mercedes Richards: Can you distinguish between the thickened part of the disk and the projection of the gas stream at phase 0.6-0.7 in the synthesized light curve?

Bisikalo: Yes, the synthetic light curve was obtained from the gas dynamic solution, where we can see the real thickenning of the circumdisk halo.

Mercedes Richards: This is very nice work! Thank you. 